\title{
Éditorial:
}

\section{La recherche fondamentale canadienne sur le vieillissement biologique: le temps du renouveau?}

Le $23^{\mathrm{e}}$ congrès annuel de l'Association canadienne de gérontologie (ACG) a eu lieu du 13 au 16 octobre 1994 à Winnipeg au Manitoba. Deux des réunions étaient consacrées aux mécanismes biologiques de base du processus de vieillissement. Les travaux alors présentés et publiés dans le présent numéro constituent l'objet de notre éditorial.

On peut raisonnablement supposer que l'être humain s'est mis à réfléchir sur son caractère mortel dès l'instant où il a été en mesure de formuler une pensée abstraite. Ce n'est cependant pas avant la fin des années 1800 qu'on a établi un cadre de travail tentant de définir le processus du vieillissement biologique en tant que phénomène réservé aux organismes multicellulaires. Dans un essai publié en 1891, August Weismann a prétendu que la mort n'était pas un attribut essentiel de la matière vivante. Il a supposé que la mort naturelle ne se produisait que dans les organismes multicellulaires et se voulait une conséquence de la séparation évolutive des cellules germinales et des cellules somatiques qui composent la grande partie des cellules d'organismes supérieurs (1). Alors que les bactéries, les levures et plusieurs autres organismes monocellulaires sont fonctionnellement immortels, des expériences effectuées vers la fin des années 1950 par Sonneborn ont démontré que certaines lignées de Paramecium monocellulaire semblent immortelles alors que d'autres ne le sont pas (2). Il semble donc que le phénomène du vieillissement (souvent appelé sénescence) se soit propagé chez les organismes monocellulaires avant même la séparation des cellules de lignée germinale et des cellules somatiques des organismes multicellulaires.

La recherche contemporaine sur le vieillissement biologique se poursuit sur un grand nombre d'organismes monocellulaires et multicellulaires, notamment sur les cellules somatiques cultivées en milieu artificiel, séparément de leur organisme hôte. Les cellules isolées et cultivées de cette façon, en dehors du corps (in vitro signifiant littéralement sous verre), semblent se rappeler leur âge par le nombre de leurs divisions lorsqu'elles sont isolées des autres cellules du corps (3). D'autres recherches ont démontré que les cellules provenant d'espèces à vie courte se divisent en moins grand nombre, in vitro, que les cellules d'organismes à vie longue (4), et ces résultats ont encouragé d'autres chercheurs à observer la façon dont le vieillissement des cellules influait sur le vieillissement de l'organisme des sujets vivant en regroupement et ont amené l'idée que le vieillissement comportait un élément génétique certain. C'est la nature de cet élément qui constituait l'objet de la première présentation du 23e congrès de l'Association 
canadienne de gérontologie.

La première oratrice de la session, Hildegard Enesco de l'Université Concordia a souligné que plus de soixante-cinq ans se sont écoulés depuis la publication des premières données scientifiques traitant de l'influence du facteur héréditaire sur la durée de la vie (5). Puisqu'il a été possible de sélectionner des organismes inférieurs à vie relativement courte sur la base de leur longévité, notamment les téphrites et les ascarides, Enesco suggère l'existence de deux catégories de gènes contribuant à la régulation de la durée de vie. La première catégorie se compose de gènes protecteurs servant à régulariser les processus physiologiques de la cellule et à la protéger des assauts physiques et biochimiques, entre autres de la radiation ultraviolette et des produits chimiques nocifs. La seconde catégorie de gènes maitrise le processus de vieillissement des cellules et on les appelle les déclencheurs de sénescence. Puisque des modifications de gènes de ces catégories peuvent avoir un effet marqué sur la durée de vie de certaines espèces animales, Enesco a soulevé l'hypothèse que le vieillissement serait dominé par quelques rares "gènes maitres" ce qui permet d'envisager la modification du processus de vieillissement par le recours à la thérapie génique (6).

Un de ces gènes porte le nom de "gêne déclencheur" (7) et code un facteur de transcription qui est une molécule régissant l'expression de plusieurs autres gènes et dont l'activité diminue avec le vieillissement de la cellule $(8,9)$. Karl Riabowol de l'Université de Calgary a démontré que la production de cet important élément cellulaire peut en fait être maîtrisée par la diminution de l'activité d'un deuxième facteur de transcription de la cellule au fur et à mesure de son vieillissement (10). Le phénomène produit un effet de cascade par lequel un simple élément de produit génique influe sur la production de plusieurs autres protéines importantes pour le développement de la cellule, empêchant par conséquent la prolongation de la croissance des cellules sénescentes. Bien que la perte de l'activité de ce facteur modifie la durée de vie in vitro des cellules isolées, on ne sait pas pour l'instant si elle aurait également une influence sur la durée de vie des animaux de laboratoire.

Dans sa définition de la sénescence, Comfort explique qu'elle «est un processus dégénératif. Lorsqu'on la mesure, on évalue la diminution de la viabilité et l'augmentation de la vulnérabilité ... elle se présente comme une hausse de la probabilité de décès reliée à l'augmentation de l'âge chronologique. L'étude de la sénescence examine un groupe de processus, différents selon les organismes, mais qui conduisent tous à l'élévation de la vulnérabilité" (11). Strehler estime nécessaire la présence de quatre facteurs dans tout changement relié à l'âge - ou à la sénescence - pour que l'on puisse parler de cause potentielle de vieillissement (12). Ce sont 1) l'universalité, 2) l'inhérence, 3) la progressivité et 4) la nocivité. Il semble que ces facteurs soient associés à l'accumulation de lésions cellulaires provenant des effets dommageables de certaines formes d'oxygènes appelées radicaux libres continuellement produits par les cellules vivantes. Jack Carlson de l'Université de Waterloo a présenté des données de son laboratoire et d'autres sources 
démontrant que les niveaux de radicaux libres augmentent lors du vieillissement et que les plus hauts niveaux d'enzymes qui protègent les cellules des radicaux sont en corrélation avec une durée de vie plus longue. En fait, dans une récente étude où les enzymes protecteurs SOD et catalase étaient produits en grande quantité dans des téphrites, on a démontré que la durée de vie de la mouche peut dans certains cas se prolonger du tiers (13), ce qui laisse croire que la diminution de l'activité des enzymes peut être une cause du vieillissement. Dans un effort pour relier les effets sur la cellule isolée aux changements de vieillissement chez l'animal, Carlson suggère que certaines cellules du corps, notamment les cellules nerveuses de l'hypothalamus qui régularisent les fonctions de la glande pituitaire sont peut-être une cible particulièrement importante d'endommagement par les radicaux libres.

La région hypothalamique du cerveau a également servi de champ d'expérience des études de vieillissement présentées par Eugenia Wang du Centre Bloomfield de recherche sur le vieillissement affilié à l'Université McGill. Étant donné que la fonction de reproduction normale repose sur plusieurs hormones produites dans la glande pituitaire et que cette glande est régularisée par des agents chimiques produits par des glandes précises de l'hypothalamus, l'équipe de Wang a examiné l'état de cellules de cette région chez des souris dont la capacité de reproduction était indubitablement à la baisse. On a découvert un processus qu'on a appelé mort programmée de la cellule où certaines cellules se dirigent vers l'autodestruction et ce processus pourrait être à l'origine, en partie du moins, de la perte des cellules observée dans cette région de l'hypothalamus. Plus récemment, l'équipe de Wang a pu observer, avec étonnement d'ailleurs, que des cellules humaines en dégénérescence développées en culture semblent résister au processus d'autodestruction programmée (14), ce qui pourrait expliquer l'augmentation de l'incidence des cancers chez plusieurs types de populations humaines d'âge avancé.

De nombreuses expériences effectuées sur les animaux permettent de croire qu'il pourrait être possible de modifier la durée de la vie humaine ou, du moins, retarder la venue des maladies reliées à l'âge. Mais avant de pouvoir déterminer si certains traitements peuvent avoir une influence sur la vitesse du vieillissement humain comme on le fait chez les animaux, il faudra développer des méthodes de mesure précises du processus du vieillissement humain puisque la vie humaine est relativement plus longue que celle des animaux utilisés couramment dans les expériences, soit les souris, les téphrites ou les ascarides (15). Toujours dans le même ordre d'idée, Robert McCulloch de l'Université de Regina a présenté une vue d'ensemble intéressante des effets du vieillissement sur la masse osseuse de la personne âgée. On savait depuis longtemps que la masse osseuse diminue chez les hommes et les femmes qui vieillissent (16), mais McCulloch a souligné le fait que l'activité physique joue un rôle important sur le maintien de la densité et de la force osseuses, particulièrement chez les personnes âgées. On a également décrit les forces et les limites des différentes techniques et des protocoles de 
recherche actuellement disponibles pour mesurer la densité osseuse avec précision.

Pendant des siècles, les philosophes ont réfléchi sur les mécanismes responsables du vieillissement biologique et plus récemment, des petits groupes de chercheurs ont étudié la question mais la quantité et la qualité d'ensemble de la recherche sur le sujet a considérablement augmenté au milieu des années 1970 au moment de la formation du National Institute on Aging des États-Unis en mai 1974 (17). La hausse de la qualité et de la quantité de recherche repose directement sur l'augmentation des fonds accordés à ce domaine, lequel concerne d'ailleurs une part de plus en plus grande de la population. Il n'existe pas encore d'institut semblable au Canada et les sommes consacrées à la recherche chez nous sont très inférieures à celles des autres pays industrialisés. Même avant les récentes réductions des budgets de recherches annoncées par le gouvernement fédéral, le Canada dépensait une part beaucoup moins importante de son produit intérieur brut en recherche et développement que tout autre grand pays industrialisé du monde. Les études révélant ces statistiques gênantes ont d'abord été effectuées par des organismes de recherche étrangers qui n'avaient aucun intérêt particulier quant à leurs résultats (18). En dépit des restrictions concernant la recherche scientifique au Canada, le pays produit tout de même des recherches de grand calibre sur le vieillissement biologique comme le démontrent les articles publiés dans ce numéro de La Revue canadienne du vieillissement. Mais leur nombre demeure nettement insuffisant compte tenu de la place qu'occupent le vieillissement et les maladies qu'il déclenche au sein de la population canadienne.

\section{Références}

1. Weismann A. Essays upon heredity and kindred biological problems. London and New York: Oxford Univ. Press (Clarendon), 1891.

2. Sonneborn TM, Rofolko M. Aging in the $P$. aurelia-multimicronucleatum complex. J. Protozool 1957; 4:21.

3. Hayflick L. The limited in vitro lifespan of human diploid cell strains. Exp. Cell Res. 1965; 37:614.

4. Martin GM, Sprague CA, Epstein CJ. Replicative life-span of cultivated human cells: Effects of donor's age, tissue and genotype. Lab. Invest. 1970: 23:86.

5. Pearl R, Pearl R de W. The ancestry of the long-lived. London: H. Milford, 1934.

6. Enesco HE. Genetic control of the aging process: a review and interpretation. La Revue canadienne du vieillissement 1996 , ce numéro.

7. Marx JL. The fos gene as "master switch". Science 1987; 237:854.

8. Seshadri T, Campisi J. Repression of $c$-fos transcription and an altered genetic program in senescent human fibroblasts. Science 1990; 247:205.

9. Riabowol K, Schiff J, Gilman MZ. Transcription factor AP-1 activity is required for initiation of DNA synthesis and is lost during cellular aging. Proc. Natl. Acad. Sci. USA. 1992; 89:157.

10. Atadja PW, Stringer KS, Riabowol KT. Loss of serum response element-binding activity and hyperphosphorylation of serum factor during cellular aging. $\mathrm{Mol}$. Cell Biol. 1994; 14:4991. 
11. Comfort A. The biology of senescence. New York: Rinehart Publishers, 1956.

12. Strehler BL. Origin and comparison of the effects of time and high energy radiations on living systems. Quart. Rev. Biol. 1959; 34:117.

13. Orr WC, Sohal RS. Extension of lifespan by overexpression of superoxide dismutase and catalase in Drosophila melanogaster. Science 1994; 263:1128.

14. Wang $\mathrm{E}$. Senescent human fibroblasts resist programmed cell death, and failure to suppress bcl2 is involved. Cancer Research 1995; 55:2285.

15. Dubina TL. Biological age as a tool for the determination of the rate of aging. Dans: Balin AK, éd. Practical handbook of human biologic age determination. Boca Raton, London, Tokyo, Ann Arbor: CRC Press, 1994.

16. Adams P, Davies GT, Sweetnam P. Osteoporosis and the effects of aging on bone mass in elderly men and women. Q. J. Medicine 1970; 39:601.

17. Strehler BL. Time, cells and aging. $2^{\mathrm{e}}$ éd. New York, San Francisco, London: Academic Press, 1977.

18. Palc, J, Anderson A. Science in Canada: underinvestment lies at the core. Nature $1988 ; 333: 719$.

Karl T. Riabowol 